\title{
Studying the environment of AT 2018cow with MUSE
}

\author{
J. D. Lyman, ${ }^{1 \star}$, L. Galbany, ${ }^{2}$ S. F. Sánchez, ${ }^{3}$ J. P. Anderson, ${ }^{4}$ H. Kuncarayakti ${ }^{5,6}$ \\ ${ }^{1}$ Department of Physics, University of Warwick, Coventry CV4 7AL, UK \\ ${ }^{2}$ Departamento de Física Teórica y del Cosmos, Universidad de Granada, E-18071 Granada, Spain \\ ${ }^{3}$ Instituto de Astronomía, Universidad Nacional Autónoma de México Circuito Exterior, Ciudad Universitaria, \\ Ciudad de México 04510, Mexico \\ ${ }^{4}$ European Southern Observatory, Alonso de Córdova 3107, Vitacura, Casilla 190001, Santiago, Chile \\ ${ }^{5}$ Tuorla Observatory, Department of Physics and Astronomy, FI-20014 University of Turku, Finland \\ ${ }^{6}$ Finnish Centre for Astronomy with ESO (FINCA), FI-20014 University of Turku, Finland
}

Accepted XXX. Received YYY; in original form ZZZ

\begin{abstract}
AT 2018cow was the nearest and best studied example of a new breed of extra-galactic, luminous and rapidly-evolving transient. Both the progenitor systems and explosion mechanisms of these rapid transients remain a mystery - the energetics, spectral signatures, and timescales make them challenging to interpret in established classes of supernovae and tidal disruption events. The rich, multi-wavelength data-set of AT 2018cow has still left several interpretations viable to explain the nature of this event. In this paper we analyse integral-field spectroscopic data of the host galaxy, CGCG 137-068, to compare environmental constraints with leading progenitor models. We find the explosion site of AT 2018cow to be very typical of core-collapse supernovae (known to form from stars with $M_{\mathrm{ZAMS}} \sim 8-25 \mathrm{M}_{\odot}$ ), and infer a young stellar population age at the explosion site of few $\times 10 \mathrm{Myr}$, at slightly sub-solar metallicity. When comparing to expectations for exotic intermediate-mass black hole (IMBH) tidal disruption events, we find no evidence for a potential host system of the IMBH. In particular, there are no abrupt changes in metallicity or kinematics in the vicinity of the explosion site, arguing against the presence of a distinct host system. The proximity of AT 2018cow to strong star-formation in the host galaxy makes us favour a massive stellar progenitor for this event.
\end{abstract}

Key words: (stars:) supernovae: individual: AT 2018cow - galaxies: individual: CGCG 137-068 - (stars:) supernovae: general - stars: massive

\section{INTRODUCTION}

Newly discovered rapidly evolving, luminous extra-galactic transients have proven a challenge to explain (e.g. Drout et al. 2014; Tanaka et al. 2016; Pursiainen et al. 2018). The discovery of such transients are a challenge for our existing understanding. Although the samples share some characteristics, studies are in their infancy and inherent diversity amongst the samples is likely to exist, this has led to such transients being named variously as FBOTs (fast and blue optical transients), FELTs (fast-evolving luminous transients) and RETs (rapidly-evolving transients), among others. Their discovery in significant numbers has been brought on by advances in cadence and depths of sky surveys in recent times. Rising typical in only a few days to luminosities exceeding typical supernovae (SNe; which typ-

^ E-mail: j.d.lyman@warwick.ac.uk (JDL) ically rise on timescales of weeks, e.g. Taddia et al. 2015), and decaying similarly rapidly poses a problem for both progenitor and explosion models, and our understanding of the final fates of stars.

Samples of such rapidly-evolving events now exist, although their nature often precludes intensive study since they must be ear-marked as interesting in real time to observe their behaviour before the onset of rapid decay - given the typical distances of the majority of events, they quickly fade below feasible observational limits. This changed with the discovery of AT 2018cow, discovered by the Asteroid Terrestrial-impact Last Alert System (ATLAS; Tonry et al. 2018) at a distance of only $\sim 61 \mathrm{Mpc}$, in the spiral galaxy CGCG 137-068 (Prentice et al. 2018).

An intensive, multi-wavelength campaign ensued for this source. As well as copious UV, optical and infra-red spectra and photometry (e.g. Prentice et al. 2018; Perley et al. 2019; Kuin et al. 2019), the source was also de- 
tected at X-ray (Rivera Sandoval et al. 2018; Margutti et al. 2019), millimeter (Ho et al. 2019) and Radio (Bietenholz et al. 2020) wavelengths. AT 2018cow displayed characteristics that matched some of the criteria for several transient models, but no single model was able to match its full behaviour. For example, the optical spectra appeared reminiscent of stripped-envelope core-collapse $\mathrm{SNe}(\mathrm{CCSNe})$ - those CCSNe with absent or tenuous hydrogen signatures - but at consistently higher temperatures and photospheric velocities than expected. Further, the tightly-constrained rapid rise to a high peak luminosity of $M_{g} \sim-20.4$ mag in only $\sim 2.5$ days (Perley et al. 2019), and subsequent power-law decay of the light-curve, do not fit well in the radioactivelypowered paradigm of CCSNe.

Additional energy sources have been suggested as significant contributors to the luminosity of AT 2018cow. Firstly, circumstellar interaction between the SN ejecta and the surrounding medium (see Chevalier \& Fransson 2017) would be an attractive additional energy-source, especially given the presence of interaction signatures in the spectra of AT 2018cow and similarities in behaviour to interacting classes such as SNe Ibn (Fox \& Smith 2019; Karamehmetoglu et al. 2019).

Central engine activity, such as in the collapsar model of CCSNe (Woosley 1993) is invoked as an additional source of energy for gamma-ray-burst $\mathrm{SNe}$ (GRB-SNe) - i.e. those $\mathrm{SNe}$ that are associated with a long-duration GRB. The accretion of stellar material onto a central black-hole (BH), formed during the SN collapse, provides additional energy injection into the SN ejecta in a strongly bi-polar orientation, eventually breaking the stellar surface to produce collimated relativistic jets. This results in the typically larger explosion energies and luminosities and temperatures of GRBSNe cf. standard CCSNe (Lyman et al. 2016; Modjaz et al. 2016; Kann et al. 2019; Taddia et al. 2019). The geometric alignment of these bi-polar jets and the Earth may be the single greatest factor in determining whether a GRB is detected associated with a collapsar SN. Nevertheless, very late time radio data can give clues as to the presence of relativistic material largely independent of the jet direction (e.g. Soderberg et al. 2010). Recent results for AT 2018cow place limits on any relativistic outflow powered by a centralengine, which, if it was produced, must have been only shortlived (Bietenholz et al. 2020).

In addition to a massive stellar origin, AT 2018cow shares a number of similarities with tidal-disruption events (TDEs). In particular the power-law decay of the light-curve is a behaviour seen in typical TDEs in the nuclei of galaxies (e.g. Arcavi et al. 2014; Holoien et al. 2016), where the central super-massive $\mathrm{BH}$ is responsible for the disruption of a main sequence star. The timescales for nuclear TDEs, however, are typically weeks-months. Perley et al. (2019) applied scaled relations to AT 2018cow in order to obtain a potential progenitor scenario involving an intermediate mass BH (IMBH; $\sim 10^{4} \mathrm{M}_{\odot}$ ) and main sequence star, whereas Kuin et al. (2019) proposed a more massive BH $\left(\sim 10^{6} \mathrm{M}_{\odot}\right)$ disrupting a low-mass white dwarf as an explanation.

The rapidly-evolving nature of transients similar to and including AT 2018cow gives a small, finite window for their direct study. However, a wealth of literature exists on probing explosive transient progenitors through analysis of their host galaxies and the explosion environments within them.
Such studies are benefiting from advents in integral-field spectroscopy (IFS), allowing the environments to be probed in ever greater details alongside their host galaxies (e.g. Lyman et al. 2018; Galbany et al. 2018; Kuncarayakti et al. 2018).

The host galaxy CGCG 137-068 has been studied at radio wavelengths, in $\mathrm{HI} 21 \mathrm{~cm}$ emission and continuum, by Roychowdhury et al. (2019) and Michałowski et al. (2019). Both studies find a ring-like morphology of the atomic Hi gas distribution in CGCG 137-068, but infer a different origin for this structure (interaction with an external galaxy, or due to resonance from the bar, respecively for the two studies.) Given the same information, the studies make conflicting interpretations on the nature of the progenitor. Roychowdhury et al. (2019) suggest the detection of Hi indicates the presence of compact star forming regions, giving a viable massive stellar progenitor route for AT 2018cow. Michałowski et al. (2019) conversely use an argument that the Hi distribution is not as asymmetric or as concentrated at the explosion site as seen in a handful of GRB-SNe hosts to argue that AT 2018cow may not have been formed by a massive star. Using ALMA data, Morokuma-Matsui et al. (2019) found the explosion site of AT 2018cow to share similarities with Type I CCSNe and the host galaxy CGCG 137-068 to be a typical star-forming dwarf in the local Universe.

Here we present Very Large Telescope/Multi Unit Spectroscopic Explorer (VLT/MUSE) integral-field spectrograph (IFS) data on CGCG 137-068 and the explosion site of AT 2018cow within, in order to investigate the nature of the progenitor system. Throughout we assume a distance of 60.9 Mpc to CGCG 137-068 based on a redshift of 0.01406 (Aguado et al. 2019) using a $\Lambda \mathrm{CDM}$ cosmology with $H_{0}=$ $70 \mathrm{~km} \mathrm{~s}^{-1} \mathrm{Mpc}^{-1}$ and $\Omega_{M}=0.3$. Distance dependant quantities do not include any uncertainty on the distance to CGCG 137-068.

\section{OBSERVATIONS AND DATA REDUCTION}

We observed CGCG 137-068, the host galaxy of AT 2018cow with MUSE (Bacon et al. 2010) mounted on UT4 of the VLT in Paranal on 2019-05-22, almost 1 year after the discovery of AT 2018cow, observations were taken as part of the Allweather MUse Supernova Integral -field of Nearby Galaxies programme (AMUSING; Galbany et al. 2016). MUSE is an integral-field unit (IFU) instrument offering seeing-limited spatially resolved spectroscopy over a $\sim 1$ arcmin field of view, large enough to cover the full extent of CGCG 137-068. The total exposure was 2805 s split over 4 exposures, which were rotated in 90 degrees steps and offset slightly to combat detector artefacts in the final data cube. The exposures were reduced and combined within the EsoREFLEX environment from ESO (Freudling et al. 2013), using MUSE pipeline version 2.6.2 and sky-residuals were removed using blank regions of the field-of-view and ZAP version 2.1 using default parameters (Soto et al. 2016). We determined the PSF FWHM to be 0.86 arcsec, as measured from isolated stars in the flatted white-light image of the cube. A reconstructed colour image of the cube is shown in Fig. 1.

As a final calibration step, we applied a flux scaling factor of 0.35 to the entire data cube. This value was found from the photometric re-calibration analysis as part of AMUS- 
ING Data Release 1 from Galbany et al. (in prep), which matches MUSE spectra to archival photometric data from the Sloan Digital Sky Survey (SDSS Fukugita et al. 1996), PanSTARRS (Tonry et al. 2012) and the Dark Energy Survey (Abbott et al. 2018) for the AMUSING++ compilation of MUSE-observed galaxies (López-Cobá et al. 2020). We expect an uncertainty on this factor comparable to the photometric accuracy of the matching (few hundredths), which is not a significant source of uncertainty for our results.

\section{DATA ANALYSIS}

For our MUSE data analysis, we used IFUANAL ${ }^{1}$ (Lyman et al. 2018). This package incorporates spectral pixel (spaxel) binning algorithms and fits stellar continuua (using StARlight, Cid Fernandes et al. 2005) and emission-lines in these spaxel bins to discern spatially-resolved properties of galaxies in IFU data. The analysis method used is detailed further in Levan et al. (2017); Lyman et al. (2018) and the documentation, ${ }^{2}$ and mirrors that done on other MUSE host galaxies (e.g. Galbany et al. 2016). Briefly, the reduced cube was dereddened by $E(B-V)=0.078 \mathrm{mag}$ (Schlafly \& Finkbeiner 2011) using an $R=3.1$ Cardelli et al. (1989) extinction law and deredshifted. Large circular masks were placed over the isolated foreground stars in the field to eliminate them from our analysis. The remaining spaxels were then binned to create distinct regions of the host which are analysed using a summed spectrum. In order to facilitate our primary emission-line analysis, we utilised a binning algorithm designed to segment HiI regions (expanded from Sánchez et al. 2012). A narrow-band $\mathrm{H} \alpha$ map (smoothed with a 0.5 pixel Gaussian filter to remove noise spikes) was constructed from the cube to determine seeds for spaxel bins as peaks in this image. These bin seeds were grown to pixels satisfying the following criteria: within $0.5 \mathrm{kpc}$ of the seed, at least 8 per cent of the seed pixel's flux and at least $2 \sigma$ above the background level of the $\mathrm{H} \alpha$ map. Where pixels lay in overlapping regions between two seeds, their assignment was to the nearest bin seed, with the distance weighted by the flux of each initial bin (prior to this assignment) to the one third power. This produced 66 bins. In addition, three circular aperture custom bins were added:

(i) The explosion site of AT 2018cow - 1.74 arcsec radius centred on explosion site, to give a $1 \mathrm{kpc}$ diameter aperture for comparison to literature samples (see Section 5).

(ii) The nucleus of CGCG 137-068 - 1 arcsecond radius centred on the nucleus, to simulate an SDSS fibre.

(iii) The integrated light of CGCG 137-068 - 12 arcsecond radius centred on the nucleus.

The location of the explosion site bin was determined from a deep, late-time William Herschel Telescope (WHT) $r$-band image, first presented in Perley et al. (2019). An affine transformation between the WHT image and a white-light image of the MUSE cube was performed with 8 sources in common using SPALIPY ${ }^{3}$. Root mean square centroid residuals

\footnotetext{
1 https://github.com/Lyalpha/ifuanal

2 https://ifuanal.readthedocs.io/en/latest/

3 https://github.com/Lyalpha/spalipy
}

were at the 0.3 pixel level, thus not contributing a significant source of uncertainty for our explosion site analyses. The integrated bin radius was selected from inspection of the flattened white-light image of the cube as the extent of detected signal from CGCG 137-068.

Each bin (including the three custom bins) was fit for the continuum using a set of Bruzual \& Charlot (2003, 2016 update) simple stellar population models from the MILES spectral library (Sánchez-Blázquez et al. 2006) with a Chabrier (2003) stellar initial mass function (IMF) from 0.1-100 $\mathrm{M}_{\odot}$. The components for the base models comprised 16 ages from $1 \mathrm{Myr}$ to $13 \mathrm{Gyr}$ for each of 4 metallicities (Z $=0.004,0.008,0.02,0.05)$. Emissions lines were fit using a series of Gaussians to obtain fluxes and line-of-sight velocities. Emission line fluxes were corrected based on the Balmer decrement assuming an expected ratio of $F_{\mathrm{H} \alpha} / F_{\mathrm{H} \beta}=2.86$ (assuming Case B recombination, Osterbrock \& Ferland 2006), which provided our estimated gas-phase extinction, $E(B-V)_{\text {gas }}$.

\section{CGCG 137-068 RESULTS}

\subsection{Stellar Continuum}

Our continuum fits for CGCG 137-068 and the explosion site of AT 2018cow are shown in Fig. 2. Based on the best fitting star-formation history used to produce these fits, the location of AT 2018cow does not appear significantly different from the overall host galaxy, containing a similar fraction of young stars, at a few-percent by mass, and being dominated by a solar-sub-solar stellar population of 10 Gyrs. We restrict our interpretation to these simple statements since the wavelength range of MUSE is limited - in particular not extending to blue wavelengths - meaning we are subject to significant uncertainty and degeneracy in our fitting, particularly when investigating the young stellar populations. The primary aim of the stellar-continuum fitting is to provide a good model continuum to subtract from our spectra to produce pure emission-line spectrum for our subsequent analysis.

Despite the caveats above, we may obtain an estimate of the total galaxy mass as this is less subject to the influence (and degeneracies) of fitting young stellar populations. From fitting the integrated spectrum of CGCG 137-068, we obtain a current stellar mass of $M_{\star}=1.74_{-0.06}^{+0.07} \times 10^{9} \mathrm{M}_{\odot}$. Quoted $1 \sigma$ uncertainties were determined from repeating the fitting on 500 realisations of the integrated spectrum, sampled from its flux and uncertainty, the adopted value is the mean of these 500 values. A host galaxy mass of $M_{\star}=1.42_{-0.29}^{+0.17} \times 10^{9} \mathrm{M}_{\odot}$ was found from spectral energy distribution (SED) fitting by Perley et al. (2019), also assuming a Chabrier (2003) stellar IMF. The two values are in good agreement $(1.8 \sigma)$ considering the slightly different distances adopted (our mass becomes $M_{\star}=1.69_{-0.06}^{+0.07} \times 10^{9} \mathrm{M}_{\odot}$ at $D_{L}=60 \mathrm{Mpc}$, the value used in Perley et al. 2019), and the different choice of stellar population bases used.

\subsection{In emission}

Emission line ratios from each of our spaxel bins (including the nucleus) lay in the region of normal emission of Hiı re- 


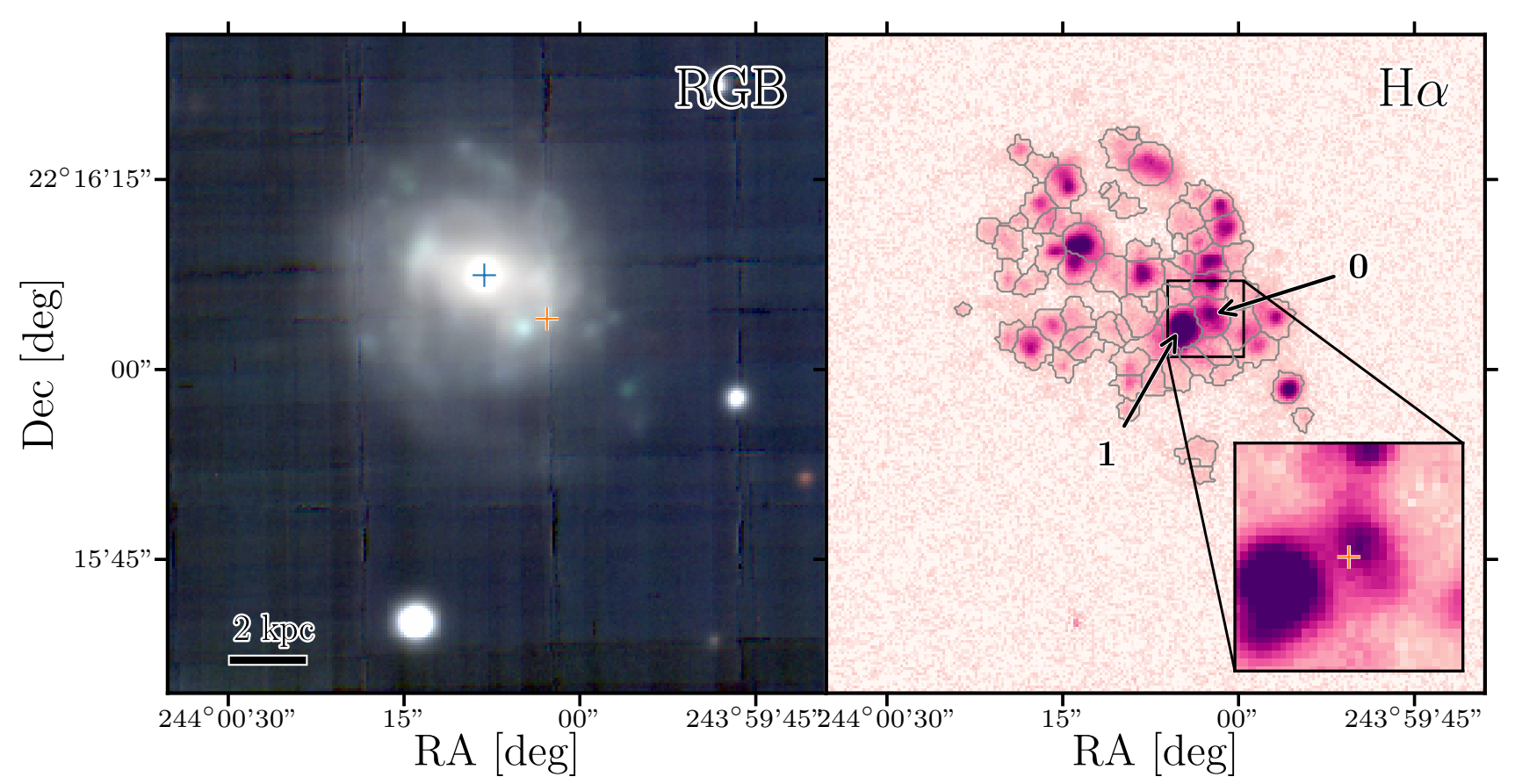

Figure 1. A false colour image (left) and a continuum-subtracted H $\alpha$ map (right) of CGCG 137-068 reconstructed from the MUSE data cube. Overlaid on the $\mathrm{H} \alpha$ map are contour lines indicating the bins used for HII region analysis - their creation is described in Section 3. The blue and orange crosses in the left panel denote the adopted locations of the nucleus of CGCG 137-068 and the explosion site of AT 2018cow, respectively. The explosion site is also indicated by the orange cross in the inset right panel. Within the inset square, which is $\sim 1.7 \mathrm{kpc}$ on a side, are shown labels for two Hil regions: Regions 0 and 1 , which we refer to throughout the analysis. These are the nearest underlying HII region to the explosion site, and the (also nearby) brightest HiI region in CGCG 137-068, respectively.

gions (Kewley et al. 2013; Sanchez 2019), i.e. the ionising radiation is dominated by the contributions of hot, young, massive stars. We thus consider the nebular emission we see in the host as being overwhelmingly due to star-formation and treat it as such. We use the calibration of Dopita et al. (2016) to determine gas-phase abundances following the recommendation of Krühler et al. (2017) for MUSE data. Portions of the continuum-subtracted spectra for our regions of interest are shown in Fig. 3 and line fluxes are presented in Table A1.

From our host nucleus extracted spectrum, we recover metallicities in agreement with SDSS-based values Morokuma-Matsui et al. (2019), namely: $Z=8.62 \pm 0.01$ (N2) and $8.72 \pm 0.03(\mathrm{O} 3 \mathrm{~N} 2)$ dex, using the calibrators of Pettini \& Pagel 2004.

Despite a somewhat regular face-on disk morphology in the continuum, a faint tidal tail is shown in the MUSE colour image extending South West, and the distribution of ongoing star-formation, as traced by $\mathrm{H} \alpha$, is quite irregular, significantly asymmetrical, and weighted in the direction of this tail (Fig. 1). This would suggest relatively recent dynamical interaction and/or gas accretion in the history of CGCG 137-068. A similar conclusion was drawn by Roychowdhury et al. (2019) based on the presence of an atomic Hi gas ring.

The point-like source to the southeast of the explosion site (Region 1) is confirmed by the MUSE data as a young, intensely star-forming HiI region, as predicted by Perley et al. (2019). Indeed, this is the most intensely star- forming region of the galaxy with $\log _{10} L_{\mathrm{H} \alpha}=39.52 \mathrm{erg} \mathrm{s}^{-1}$ In our data we discover a further relatively bright HII region almost directly underlying the explosion site (Region 0 ), which was obscured in earlier data containing emission from AT $2018 \mathrm{cow}$, having $\log _{10} L_{\mathrm{H} \alpha}=39.13 \mathrm{erg} \mathrm{s}^{-1}$, about 100 times that of Orion (Kennicutt 1984). The total H $\alpha$ luminosity of CGCG 137-068, as determined from the integrated bin, is $\log _{10} L_{\mathrm{H} \alpha}=40.37 \mathrm{erg} \mathrm{s}^{-1}$, making it wholly unremarkable in the local Universe.

Converting from $\mathrm{H} \alpha$ luminosity to SFR using the calibration of Kennicutt (1998), we obtain a total $\mathrm{SFR}_{\mathrm{H} \alpha}=$ $0.19 \mathrm{M}_{\odot} /$ year, in excellent agreement with the value of $0.22_{-0.04}^{+0.03} \mathrm{M}_{\odot} /$ year inferred by Perley et al. (2019) from SED fitting of CGCG 137-068.

\subsection{Explosion site of AT 2018cow}

The adopted explosion site of AT 2018cow lies $0.45 \mathrm{arcsec}$ ( $\sim 130$ pc at the distance of CGCG 137-068, in projection) from the peak of Region 0 , and $2 \operatorname{arcsec}$ from Region 1 $(\sim 570 \mathrm{pc})$. Although Region 1 is the most intensely starforming region of the galaxy, it does not differ significantly from Region 0 (Table 1), perhaps being slightly younger and metal-rich. Thus the ambiguity in the parent HiI region of AT 2018cow does not affect significantly the environmental constraints on the progenitor. (Any such constraints from the HiI regions assume the progenitor was formed recently, 


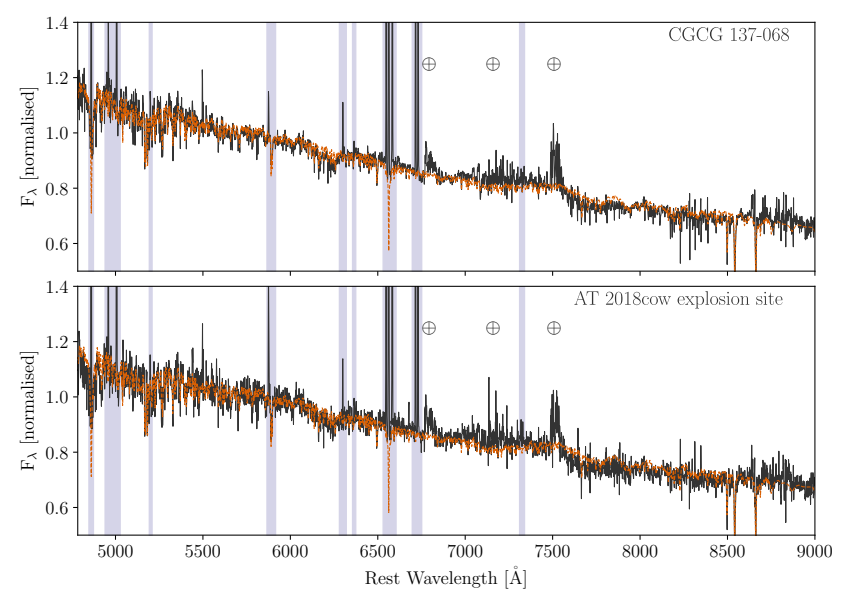

Figure 2. Top: Stellar population fit from STARLight for CGCG 137-068 showing extracted MUSE spectrum (black) with model continuum fit (orange, dashed). Shaded regions were masked from the fitting procedure. Bottom: As above but for the explosion site of AT $2018 \mathrm{cow}$. This region appears to closely resemble the integrated spectrum, making the explosion site typical of the host galaxy. Spectra are normalised to the flux in the range $5590-5680 \AA$.

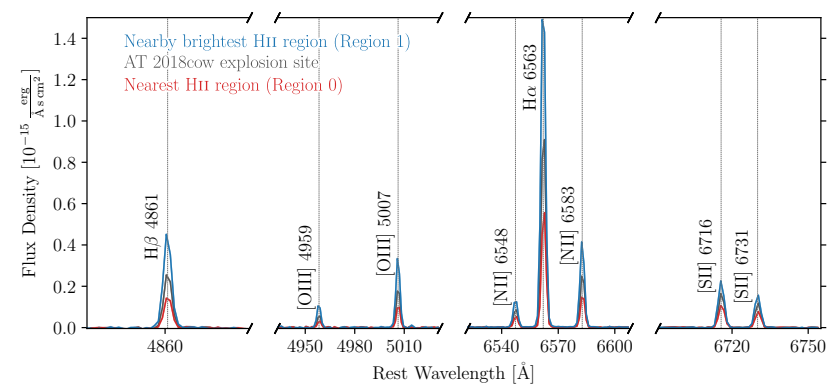

Figure 3. Continuum-subtracted spectra extracted at the regions of interest in the analysis (see Fig. 1). Spectra have been cut to highlight the strong-lines of interest. Line fluxes are given in Table A1.

and thus coeval with the ongoing star-formation of these regions, see Section 5.)

The presence of HiI regions almost exactly underlying the explosion site hints towards a causal link between AT 2018cow and these regions - the spatial coverage of similarly bright regions as compared to the continuum distribution (Fig. 1) is low.

The largely uncertain nature of the progenitor AT 2018cow, and its spectral similarity to some interacting SNe around maximum light (Fox \& Smith 2019) merits a search for late-time nebular or interaction emission-line signatures at its explosion site. A manual inspection of the continuum-subtracted spectrum revealed no sources of flux that we could not ascribe to normal HiI region emission.

We show the locations of these regions in the cumulative distribution of star-formation throughout CGCG 137-068 in Fig. 4. The host galaxy HiI regions contribute a weight to the cumulative distribution given by their (Balmer decrementcorrected) $\mathrm{H} \alpha$ luminosity. The distributions thus show the

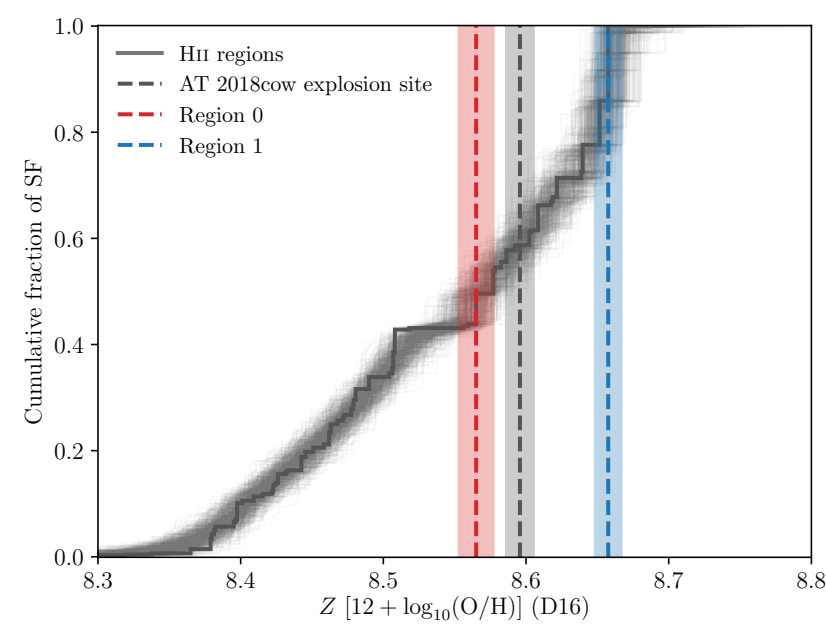

Figure 4. Cumulative distributions showing the distributions of star-formation as a function of metallicity in CGCG 137-068 using the indicator of Dopita et al. (2016). The HII regions contribute a weight to the distribution given by their $\mathrm{H} \alpha$ luminosity, making the distribution a cumulative fraction of ongoing star formation (rather than just raw counting of HiI regions). 500 realisations are shown assuming Gaussian statistical uncertainties on the metallicity and $\mathrm{H} \alpha$ luminosity weighting.

fraction of stars being formed at that metallicity or less, giving the ZNCR statistic introduced in Lyman et al. (2018). Although we see the choice of metallicity indicator has a noticable impact on the distributions, and in particular the position of Region 1 within the respective distributions, the explosion site of AT 2018cow consistently has a ZNCR $0.5-0.6$, indicating it is a typical metallicity for stars being formed in CGCG 137-068.

\section{COMPARISON TO OTHER TRANSIENTS ENVIRONMENTS}

Here we assess our findings on the environment of AT 2018cow in the context of known and expected environment properties for plausible progenitor explanations.

\subsection{Massive star progenitor}

If we take AT 2018cow to have arisen from a massive stellar explosion, we may expect some similarity to the environments of CCSNe. Indeed, AT 2018cow being hosted by a later-type galaxy and located in a region of ongoing starformation, seems to meet the expectations of a young progenitor. For a more quantitative comparison we use the PISCO sample of SN environment properties from (Galbany et al. 2018). The advantage of using this IFU-based CCSN environment sample is that we are able to compare directly with the sample physical size aperture $(1 \mathrm{kpc})$. We compare AT 2018cow to stripped-envelope SN (SESN) sample (i.e. those CCSNe showing absent or tenuous $\mathrm{H}$ features) and interacting SNe IIn owing to having the closest similarities in terms of empirical classification with AT 2018cow (e.g. Perley et al. 2019; Fox \& Smith 2019) in Fig. 5. The explosion site of AT 2018cow appears almost exactly half way along the 
Table 1. Strong-emission line results for regions of interest in CGCG 137-068 (regions defined in Fig. 1). Uncertainties quoted are statistical only.

\begin{tabular}{lcccc}
\hline Location & $\begin{array}{c}E(B-V)_{\text {gas }} \\
\mathrm{mag}\end{array}$ & $\begin{array}{c}L(\mathrm{H} \boldsymbol{\alpha}) \\
{\left[\log _{10} \mathrm{erg} \mathrm{s}^{-1}\right]}\end{array}$ & $\begin{array}{c}\mathrm{EW}(\mathrm{H} \boldsymbol{\alpha}) \\
{[\AA] \AA}\end{array}$ & $\begin{array}{c}Z^{\mathrm{a}} \\
12+\log _{10}(\mathrm{O} / \mathrm{H})\end{array}$ \\
\hline AT 2018cow explosion site & 0.23 & $39.33 \pm 0.01$ & $78.7 \pm 2.1$ & $8.60 \pm 0.01$ \\
Region 0 & 0.26 & $39.13 \pm 0.01$ & $65.9 \pm 1.9$ & $8.57 \pm 0.01$ \\
Region 1 & 0.19 & $39.52 \pm 0.01$ & $111.3 \pm 2.9$ & $8.66 \pm 0.01$ \\
CGCG 137-068 (nucleus) & 0.27 & $38.88 \pm 0.01$ & $22.3 \pm 0.7$ & $8.65 \pm 0.02$ \\
CGCG 137-068 (integrated) & 0.19 & $40.37 \pm 0.01$ & $33.9 \pm 0.9$ & $8.53 \pm 0.01$
\end{tabular}

${ }^{\text {a }}$ Gas-phase abundance measured in the scale of Dopita et al. (2016).

SN metallicity distributions, and lies towards the upper end of the $\mathrm{H} \alpha \mathrm{EW}$ distribution (indicating a relatively younger environment cf. the median SESN or SN IIn in these comparison samples). However, given the comparison distributions span relative wide-ranges, and we are comparing them with a single object, making any firm inferences on the progenitor of AT $2018 \mathrm{cow}$ from these plots appears to be frivolous.

It is of interest to compare AT 2018cow with the environments of SNe Ibn (i.e. hydrogen-poor SNe with signature of narrow helium lines). AT 2018cow has been discussed as sharing similarities to SN Ibn both spectroscopically (Fox \& Smith 2019) and photometrically (Karamehmetoglu et al. 2019). The progenitors of SN Ibn are currently debated. In the remainder of the paragraph we determine what we may learn about SNe Ibn if we assume AT 2018cow as being a member of the class - in the opposing case such arguments would be applicable to only the progenitor of AT 2018cow and we of course cannot infer any extension to SNe Ibn progenitors. The favoured model of Wang \& Li (2019) for the rapidly-rising and luminous SN Ibn PS15dpn invokes an interaction $+{ }^{56} \mathrm{Ni}$ powered CCSN explosion. Given their inferred ejecta mass $\left(\sim 13 \mathrm{M}_{\odot}\right)$ is significantly above ejected masses from typical stripped-envelope SNe (Lyman et al. 2016), the authors suggest a Wolf-Rayet (WR) progenitor for this SN Ibn. WR stars are amongst the most massive (and thus short-lived - few Myr) stars. Neither Region 0 or 1 exhibit the properties of WR regions: i) our $\mathrm{H} \alpha$ EW measurements would place the regions at ages of tens of Myr (Lyman et al. 2016; Xiao et al. 2019), based on comparison with models of stellar populations including multiplicity from the Binary Population and Spectral Synthesis code (Eldridge et al. $2017)$; ii) we find no evidence in the continuum-subtracted spectra for emission lines either indicating directly the presence of WR stars (e.g. the red bump, C III/IV N III/IV) or very young SPs (He I 14922). ${ }^{4}$ We therefore argue against a WR origin for AT $2018 \mathrm{cow}$. These arguments also hold for the suggestion of pulsational pair-instability SNe (Woosley et al. 2007) as the progenitors for SNe Ibn (Karamehmetoglu et al. 2019), since these SNe are expected to be also caused by extremely massive stars $\left(\mathrm{M}_{\mathrm{ZAMS}} \sim 100 \mathrm{M}_{\odot}\right)$. IFS environment studies of samples of SNe Ibn are in progress to address questions about their progenitors, and thus the comparison to AT 2018cow, more thoroughly.

The maintenance of very high temperatures, its large photospheric velocities (Prentice et al. 2018; Perley et al. 2019) and large X-ray flux (Rivera Sandoval et al. 2018;

\footnotetext{
${ }^{4} 5 \sigma$ line fluxes were $\sim 0.7-1 \times 10^{-17} \mathrm{erg} \mathrm{s}^{-1} \mathrm{~cm}^{-2}$ in the regions
} surrounding AT 2018cow.
Kuin et al. 2019) make AT 2018cow appear reminiscent of $\mathrm{SNe}$ associated with additional energy injection, such as engine-driven events giving rise to long-duration gammaray bursts (LGRBs). Such GRB-SNe, and LGRBs in general, preferentially inhabit younger environments compared to other CCSN types (e.g. Fruchter et al. 2006; Kelly et al. 2014; Lyman et al. 2017). Indeed the host galaxy and specific explosion location of AT 2018cow is remarkably similar to two of the best studied low-redshift GRBSNe: GRB 980425/SN 1998bw (Krühler et al. 2017) and GRB 100316D/SN 2010bh (Izzo et al. 2017). Both were located coincident with strong star-forming regions and close to $(<1 \mathrm{kpc})$ the most intensely star-forming region of their hosts. There is evidence that LGRBs are subject to a metallicity cut-off, above which their production is suppressed, although the location of this cut-off is debated and may be higher than previously determined, at roughly solar (Perley et al. 2016). Given our determined metallicity for the explosion site is slightly sub-solar, there does not appear great tension with this cut-off.

The high peak luminosity of AT 2018cow makes it comparable to super-luminous SNe (SLSNe; e.g. Gal-Yam 2012; Inserra et al. 2013), which are expected to arise from particularly massive stellar explosions with additional energy input thought to arise from magnetar spin-down or circumstellar interaction. Their host galaxies and environments share a number of similarities with those of LGRBs (e.g. Lunnan et al. 2014; Angus et al. 2016; Lyman et al. 2017), being generally low-mass and compact. Although no data are available for a direct comparison in our Dopita et al. (2016) metallicities, a number of studies have noted the strong metal-aversion of SLSN production (e.g. Chen et al. 2017; Schulze et al. 2018), indicating a threshold of roughly half-solar for their environments. For such a cut-off, our almost solar metallicity determined at the explosion site of AT 2018cow would place it in tension with this progenitor interpretation, although exceptions exist (e.g. SN 2017egm Nicholl et al. 2017).

Environmental arguments have been made to suggest AT 2018cow was not a massive star based solely on the comparison to GRB-SNe environments by observing the form of Hi gas distributions of the respective host galaxies Michałowski et al. (2019). However, GRB-SNe production is a relatively unknown process that occurs in only a few percent of all CCSNe (e.g. Graham \& Schady 2016) and indeed could have a causal link or be enhanced with the presence of relatively pristine gas inflows in the local Universe, given the metallicity cut-off seen for LGRB progenitors. Our discovery of strong HiI region emission located at the explosion site of AT 2018cow appears a strong means to link the progenitor 
with ongoing star-formation, and thus young (massive) stellar populations. This in contrast to inference from observing cold inflows of neutral gas, which provide an even more indirect indicator of current star-formation. We note however that it is very difficult to make conclusive statements on a single object, given almost any location in a galaxy will have a significant line-of-sight population of old stars.

\section{$5.2 \quad$ Tidal-disruption event}

Other explanations for AT 2018cow have been proposed that may not involve a massive star at all. One such scenario is the tidal-disruption of a main-sequence star or white dwarf by an IMBH (Perley et al. 2019; Kuin et al. 2019).

In the main-sequence TDE scenario (Perley et al. 2019), a BH mass of $\sim 10^{4.3} \mathrm{M}_{\odot}$ is required. Such an IMBH would be expected to be located within a massive stellar cluster. For the case of a young parent cluster, we do indeed find a star-forming region underlying AT 2018cow, however the explosion site is significantly offset from the peak of this source in both the white-light image of our MUSE cube and the centroid of the $\mathrm{H} \alpha$ emission, where one would expect the massive IMBH to settle towards rapidly. For an older, globular cluster (GC) host, the hosting GC would lie at the upper end of the GC luminosity function (Harris 1996). A magnitude of the host of $M_{V}, \sim-9$ to -11 mag follows from both extending the M- $\sigma$ relation (e.g. Gültekin et al. 2009) to IMBH masses, and looking at the luminosities of GCs with signs of IMBHs (e.g. Noyola et al. 2010; Lützgendorf et al. 2013; Feldmeier et al. 2013). At the distance of CGCG 137-068 this would appear as a $m_{V} \sim 23-25 \mathrm{mag}$ source at the explosion site, and should be detectable in high-resolution imaging of the site.

An alternate scenario, involving a more massive $\mathrm{BH}$ $\sim 10^{5-6} \mathrm{M}_{\odot}$ and a low-mass white dwarf was presented by Kuin et al. (2019). Although white dwarfs may form at a few $\times 10^{7}$ yrs after star-formation (Portegies Zwart et al. 2007), i.e. comparable to the age we infer for the underlying HiI at the explosion site of AT 2018cow), these are the most massive white-dwarfs - low-mass white-dwarfs will form much later. In this case the co-location with star-formation is a coincidence in this scenario. This $\mathrm{BH}$ required mass is comparable to that of a massive GC itself, and a dwarf satellite galaxy of CGCG 137-068 may be a more promising BH-host system. Notwithstanding the lack of spatial coincidence we find between AT 2018cow and the peak of any underlying source, we searched in our data for existence of a distinct system in the vicinity. In Fig. 6 we show the results of fitting individual spaxels in a region surrounding the explosion site of AT 2018cow to look for departures from smoothly varying behaviour in line-of-sight velocity of the gas and stars, and the gas-phase metallicity. We find no evidence for a distinct velocity component in the maps and the line profiles are well modelled by single Gaussians (Fig. 3), arguing against the presence of any satellite galaxy at this location. We also note the metallicity is smoothly varying over this region, whereas a satellite galaxy may be expected to have a different metallicity profile.
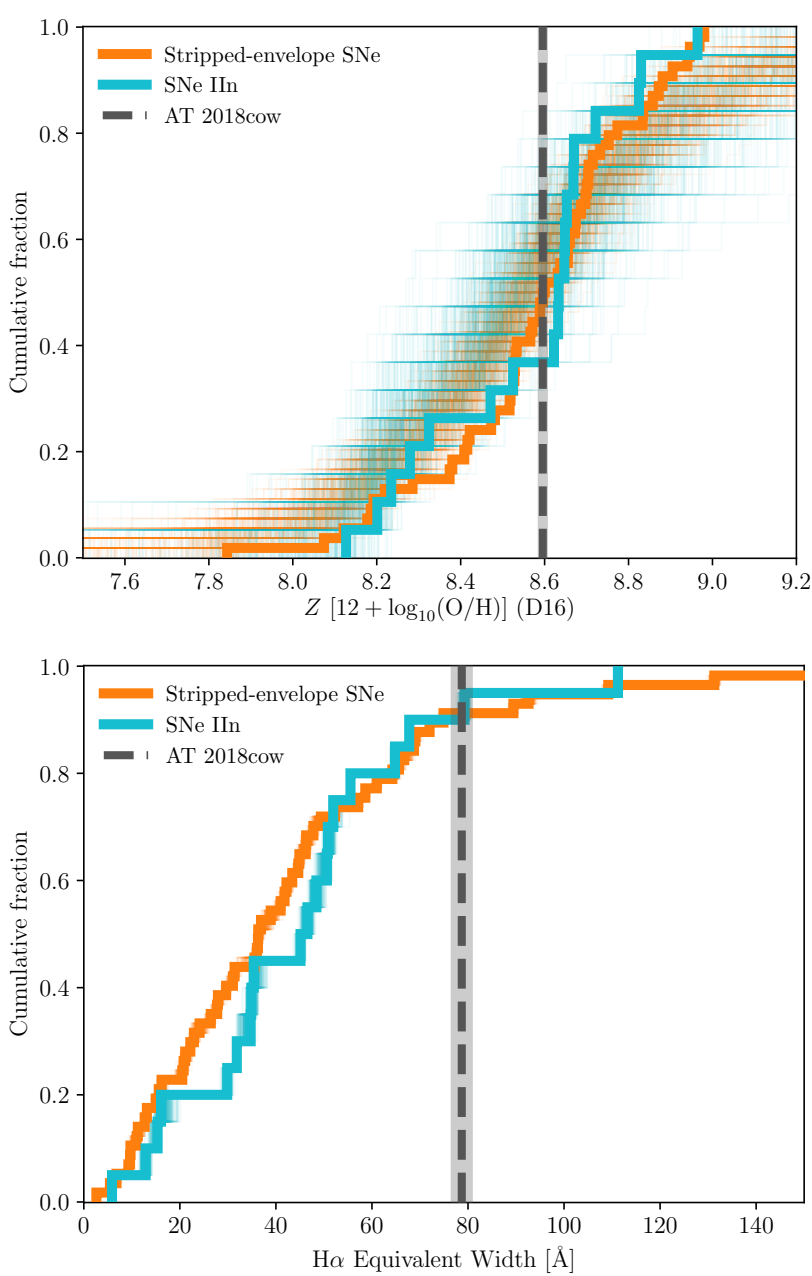

Figure 5. Cumulative distributions of metallicity (top) and $\mathrm{H} \alpha \mathrm{EW}$ (bottom) for stripped-envelope SN environments in the PISCO sample (Galbany et al. 2018). Distributions are shown following Fig. 4. (The 'spreading' of the re-sampled metallicity distributions owes to the relatively large uncertainties compared to the range of values). The explosion site of AT 2018cow is indicated on each plot.

\section{CONCLUSIONS}

On the balance of evidence presented by our MUSE data, we would favour a young (and therefore likely to be massive) progenitor for AT 2018 cow. We find the transient exploded in close proximity to the most intensely star-forming region of the galaxy and coincident with an underlying HII region. The environment appears typical of other massive star progenitor CCSNe explosion sites based on optical diagnostics, indicating a progenitor age of tens of Myrs. Alternative scenarios, particularly those involving tidal disruptions by IMBHs are less favoured based on a lack of evidence for a massive host system at the site that could plausibly host the IMBH, although deep, high-resolution imaging is required to place meaningful limits on globular clusters. Our deep synthesised optical image of CGCG 137-068 indicates tidal tails and the $\mathrm{H} \alpha$ image indicates asymmetry in the spatial distribution of star-formation, indicative of recent merger history for the galaxy. 


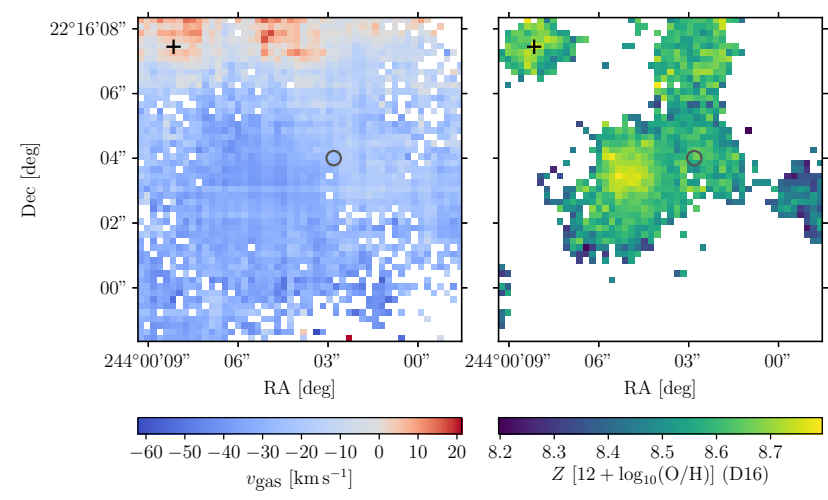

Figure 6. The velocity (left) and metallicity (right) of gas in CGCG 137-068 surrounding the explosion site of AT 2018cow. The velocity was determined from the peak of a Gaussian fitted to $\mathrm{H} \alpha$ and is shown relative to the adopted redshift of CGCG 137-068. White pixels indicate regions where the signal-to-noise ratio of the emission lines prevented the fitting procedure from converging. Given these smoothly evolving maps, we find no evidence of a distinct satellite source underlying AT 2018cow, which may have appeared as distinct in the velocity and metallicity maps. The explosion site of AT 2018cow and the nucleus of CGCG 137-068 are indicated by a circle and cross in each panel, respectively.

A comparison of our environmental results for the very local and fortuitous AT 2018cow will need to be considered alongside statistical analyses of the environments of more distant similar transient samples in order to elucidate the progenitors and diversity of these fast, luminous transients.

\section{ACKNOWLEDGEMENTS}

We thank Dan Perley for kindly providing deep WHT imaging of AT 2018cow from which to perform relative astrometry. Klaas Wiersema and Elizabeth Stanway are thanked for useful discussions. JDL acknowledges support from STFC via grant $\mathrm{ST} / \mathrm{P} 000495 / 1$. LG was funded by the European Union's Horizon 2020 research and innovation programme under the Marie Skłodowska-Curie grant agreement No. 839090. This work has been partially supported by the Spanish grant PGC2018-095317-B-C21 within the European Funds for Regional Development (FEDER). SFS thanks for the support of a CONACYT grant CB-285080 and FC-201601-1916, and funding from the PAPIIT-DGAPA-IN100519 (UNAM) project. We acknowledge the usage of the HyperLeda database (http://leda.univ-lyon1.fr) Based on observations collected at the European Organisation for Astronomical Research in the Southern Hemisphere under ESO programme 0103.D-0440(A). This research made use of Astropy, ${ }^{5}$ a community-developed core Python package for Astronomy (Astropy Collaboration et al. 2013, 2018).

\section{REFERENCES}

Abbott T. M. C., et al., 2018, ApJS, 239, 18

5 http://www.astropy.org
Aguado D. S., et al., 2019, ApJS, 240, 23

Angus C. R., Levan A. J., Perley D. A., Tanvir N. R., Lyman J. D., Stanway E. R., Fruchter A. S., 2016, MNRAS, 458, 84 Arcavi I., et al., 2014, ApJ, 793, 38

Astropy Collaboration et al., 2013, A\&A, 558, A33

Astropy Collaboration et al., 2018, AJ, 156, 123

Bacon R., et al., 2010, The MUSE second-generation VLT instrument. p. 773508 , doi:10.1117/12.856027

Bietenholz M. F., et al., 2020, MNRAS, 491, 4735

Bruzual G., Charlot S., 2003, MNRAS, 344, 1000

Cardelli J. A., Clayton G. C., Mathis J. S., 1989, ApJ, 345, 245

Chabrier G., 2003, PASP, 115, 763

Chen T.-W., Smartt S. J., Yates R. M., Nicholl M., Krühler T., Schady P., Dennefeld M., Inserra C., 2017, MNRAS, 470, 3566

Chevalier R. A., Fransson C., 2017, Thermal and Nonthermal Emission from Circumstellar Interaction. p. 875, doi:10.1007/978-3-319-21846-5_34

Cid Fernandes R., Mateus A., Sodré L., Stasińska G., Gomes J. M., 2005, MNRAS, 358, 363

Dopita M. A., Kewley L. J., Sutherland R. S., Nicholls D. C., 2016, Ap\&SS, 361, 61

Drout M. R., et al., 2014, ApJ, 794, 23

Eldridge J. J., Stanway E. R., Xiao L., McClelland L. A. S., Taylor G., Ng M., Greis S. M. L., Bray J. C., 2017, Publ. Astron. Soc. Australia, 34, e058

Feldmeier A., et al., 2013, A\&A, 554, A63

Fox O. D., Smith N., 2019, MNRAS, 488, 3772

Freudling W., Romaniello M., Bramich D. M., Ballester P., Forchi V., García-Dabló C. E., Moehler S., Neeser M. J., 2013, A\&A, 559, A96

Fruchter A. S., et al., 2006, Nature, 441, 463

Fukugita M., Ichikawa T., Gunn J. E., Doi M., Shimasaku K., Schneider D. P., 1996, AJ, 111, 1748

Gal-Yam A., 2012, Science, 337, 927

Galbany L., et al., 2016, MNRAS, 455, 4087

Galbany L., et al., 2018, ApJ, 855, 107

Graham J. F., Schady P., 2016, ApJ, 823, 154

Gültekin K., et al., 2009, ApJ, 698, 198

Harris W. E., 1996, AJ, 112, 1487

Ho A. Y. Q., et al., 2019, ApJ, 871, 73

Holoien T. W. S., et al., 2016, MNRAS, 463, 3813

Inserra C., et al., 2013, ApJ, 770, 128

Izzo L., et al., 2017, MNRAS, 472, 4480

Kann D. A., et al., 2019, A\&A, 624, A143

Karamehmetoglu E., et al., 2019, arXiv e-prints, p. arXiv:1910.06016

Kelly P. L., Filippenko A. V., Modjaz M., Kocevski D., 2014, ApJ, 789, 23

Kennicutt R. C. J., 1984, ApJ, 287, 116

Kennicutt Robert C. J., 1998, ARA\&A, 36, 189

Kewley L. J., Maier C., Yabe K., Ohta K., Akiyama M., Dopita M. A., Yuan T., 2013, ApJ, 774, L10

Krühler T., Kuncarayakti H., Schady P., Anderson J. P., Galbany L., Gensior J., 2017, A\&A, 602, A85

Kuin N. P. M., et al., 2019, MNRAS, 487, 2505

Kuncarayakti H., et al., 2018, A\&A, 613, A35

Levan A. J., et al., 2017, ApJ, 848, L28

López-Cobá C., et al., 2020, AJ, 159, 167

Lunnan R., et al., 2014, ApJ, 787, 138

Lützgendorf N., et al., 2013, A\&A, 552, A49

Lyman J. D., Bersier D., James P. A., Mazzali P. A., Eldridge J. J., Fraser M., Pian E., 2016, MNRAS, 457, 328

Lyman J. D., et al., 2017, MNRAS, 467, 1795

Lyman J. D., et al., 2018, MNRAS, 473, 1359

Margutti R., et al., 2019, ApJ, 872, 18

Michałowski M. J., et al., 2019, A\&A, 627, A106

Modjaz M., Liu Y. Q., Bianco F. B., Graur O., 2016, ApJ, 832, 108 
Morokuma-Matsui K., et al., 2019, arXiv e-prints, p. arXiv:1906.05446

Nicholl M., Berger E., Margutti R., Blanchard P. K., Guillochon J., Leja J., Chornock R., 2017, ApJ, 845, L8

Noyola E., Gebhardt K., Kissler-Patig M., Lützgendorf N., Jalali B., de Zeeuw P. T., Baumgardt H., 2010, ApJ, 719, L60

Osterbrock D. E., Ferland G. J., 2006, Astrophysics of gaseous nebulae and active galactic nuclei

Perley D. A., et al., 2016, ApJ, 817, 8

Perley D. A., et al., 2019, MNRAS, 484, 1031

Pettini M., Pagel B. E. J., 2004, MNRAS, 348, L59

Portegies Zwart S. F., McMillan S. L. W., Makino J., 2007, MNRAS, 374, 95

Prentice S. J., et al., 2018, ApJ, 865, L3

Pursiainen M., et al., 2018, MNRAS, 481, 894

Rivera Sandoval L. E., Maccarone T. J., Corsi A., Brown P. J., Pooley D., Wheeler J. C., 2018, MNRAS, 480, L146

Roychowdhury S., Arabsalmani M., Kanekar N., 2019, MNRAS, 485, L93

Sanchez S. F., 2019, arXiv e-prints, p. arXiv:1911.06925

Sánchez-Blázquez P., et al., 2006, MNRAS, 371, 703

Sánchez S. F., et al., 2012, A\&A, 546, A2

Schlafly E. F., Finkbeiner D. P., 2011, ApJ, 737, 103

Schulze S., et al., 2018, MNRAS, 473, 1258

Soderberg A. M., et al., 2010, Nature, 463, 513

Soto K. T., Lilly S. J., Bacon R., Richard J., Conseil S., 2016, MNRAS, 458, 3210

Taddia F., et al., 2015, A\&A, 574, A60

Taddia F., et al., 2019, A\&A, 621, A71

Tanaka M., et al., 2016, ApJ, 819, 5

Tonry J. L., et al., 2012, ApJ, 750, 99

Tonry J. L., et al., 2018, PASP, 130, 064505

Wang S.-Q., Li L., 2019, arXiv e-prints, p. arXiv:1905.12623

Woosley S. E., 1993, ApJ, 405, 273

Woosley S. E., Blinnikov S., Heger A., 2007, Nature, 450, 390

Xiao L., Galbany L., Eldridge J. J., Stanway E. R., 2019, MNRAS, 482,384

\section{APPENDIX A: EMISSION LINE FLUXES}

For completeness, individual emission line flux measurements from continuum-subtracted spectra for regions of interest (see Section 3) are presented in Table A1.

This paper has been typeset from a $\mathrm{T}_{\mathrm{E}} \mathrm{X} / \mathrm{LAT}_{\mathrm{E}} \mathrm{X}$ file prepared by the author. 
Table A1. Emission line fluxes for regions of interest in CGCG 137-068. Fluxes are not corrected for intrinsic extinction, but have been corrected for foreground Galactic extinction (Section 3). Units are $10^{-15} \mathrm{erg} \mathrm{s}^{-1} \mathrm{~cm}^{-2}$. Uncertainties quoted are statistical only and limits at $3 \sigma$.

\begin{tabular}{lccccccc}
\hline Location & $\mathrm{H} \beta$ & {$[\mathrm{OIII}] 4959$} & {$[\mathrm{OIII}] 5007$} & $\mathrm{HeI} 5876$ & {$[\mathrm{OI}] 6300$} & {$[\mathrm{OI}] 6364$} & {$[\mathrm{NII}] 6548$} \\
\hline AT 2018cow explosion site & $1.69 \pm 0.06$ & $0.35 \pm 0.03$ & $1.16 \pm 0.05$ & $0.18 \pm 0.02$ & $0.08 \pm 0.02$ & $<0.04$ & $0.44 \pm 0.01$ \\
Region 0 & $2.62 \pm 0.07$ & $0.61 \pm 0.04$ & $1.86 \pm 0.06$ & $0.25 \pm 0.03$ & $0.07 \pm 0.02$ & $<0.04$ & $0.63 \pm 0.02$ \\
Region 1 & $1.07 \pm 0.05$ & $0.21 \pm 0.03$ & $0.71 \pm 0.04$ & $0.11 \pm 0.02$ & $0.07 \pm 0.02$ & $<0.03$ & $0.29 \pm 0.01$ \\
CGCG 137-068 (nucleus) & $0.60 \pm 0.05$ & $<0.10$ & $0.20 \pm 0.04$ & $<0.07$ & $<0.05$ & $<0.03$ & $0.19 \pm 0.01$ \\
CGCG 137-068 (integrated) & $18.53 \pm 0.80$ & $3.37 \pm 0.48$ & $11.15 \pm 0.68$ & $1.64 \pm 0.37$ & $1.74 \pm 0.29$ & $0.63 \pm 0.19$ & $5.25 \pm 0.24$ \\
\hline Location & $\mathrm{H} \alpha$ & {$[\mathrm{NII}] 6583$} & HeI 6678 & {$[\mathrm{SII}] 6716$} & {$[\mathrm{SII}] 6731$} & ArIII 7136 & {$[\mathrm{SIII}] 9067$} \\
\hline AT 2018cow explosion site & $4.84 \pm 0.03$ & $1.28 \pm 0.02$ & $0.80 \pm 0.02$ & $0.55 \pm 0.01$ & $0.27 \pm 0.01$ & $0.06 \pm 0.01$ & $0.08 \pm 0.01$ \\
Region 0 & $7.49 \pm 0.04$ & $1.88 \pm 0.03$ & $1.00 \pm 0.02$ & $0.70 \pm 0.02$ & $0.45 \pm 0.02$ & $0.08 \pm 0.01$ & $0.14 \pm 0.02$ \\
Region 1 & $3.06 \pm 0.03$ & $0.84 \pm 0.02$ & $0.57 \pm 0.01$ & $0.39 \pm 0.01$ & $0.17 \pm 0.01$ & $0.04 \pm 0.01$ & $0.05 \pm 0.01$ \\
CGCG 137-068 (nucleus) & $1.71 \pm 0.03$ & $0.55 \pm 0.02$ & $0.32 \pm 0.01$ & $0.22 \pm 0.01$ & $0.07 \pm 0.01$ & $<0.03$ & $<0.04$ \\
CGCG 137-068 (integrated) & $52.99 \pm 0.43$ & $15.40 \pm 0.33$ & $11.61 \pm 0.31$ & $7.92 \pm 0.28$ & $2.90 \pm 0.19$ & $0.61 \pm 0.18$ & $0.67 \pm 0.21$ \\
\hline
\end{tabular}

